\title{
Essential Oils, A New Horizon in Combating Bacterial Antibiotic Resistance
}

\author{
Polly Soo Xi Yap ${ }^{1}$, Beow Chin Yiap², Hu Cai Ping ${ }^{3}$ and Swee Hua Erin Lim²,*
}

${ }^{1}$ School of Postgraduate Studies and Research, International Medical University, No. 126, Jalan Jalil Perkasa 19, Bukit
Jalil, 57000 Kuala Lumpur, Malaysia; ${ }^{2}$ School of Pharmacy, Department of Life Sciences, International Medical Uni-
versity, No. 126, Jalan Jalil Perkasa 19, Bukit Jalil, 57000 Kuala Lumpur, Malaysia; ${ }^{3}$ School of Health Sciences, De-
partment of Chinese Medicine, International Medical University, No. 126, Jalan Jalil Perkasa 19, Bukit Jalil, 57000
Kuala Lumpur, Malaysia

\begin{abstract}
For many years, the battle between humans and the multitudes of infection and disease causing pathogens continues. Emerging at the battlefield as some of the most significant challenges to human health are bacterial resistance and its rapid rise. These have become a major concern in global public health invigorating the need for new antimicrobial compounds. A rational approach to deal with antibiotic resistance problems requires detailed knowledge of the different biological and non-biological factors that affect the rate and extent of resistance development. Combination therapy combining conventional antibiotics and essential oils is currently blooming and represents a potential area for future investigations. This new generation of phytopharmaceuticals may shed light on the development of new pharmacological regimes in combating antibiotic resistance. This review consolidated and described the observed synergistic outcome between essential oils and antibiotics, and highlighted the possibilities of essential oils as the potential resistance modifying agent.
\end{abstract}

Keywords: Antibiotic resistance, combination therapy, essential oils, resistance modifying agents.

\section{INTRODUCTION}

Antibiotic therapy is one of the most important therapies used for fighting infectious diseases and has tremendously enhanced the health aspects of human life since its introduction. Despite the advancements in this therapy, we still live in an era where incidents of antibiotic resistant infections are alarmingly on rise [1]. The significance of the role of antibiotics in nature remains unfounded due to the responses of bacteria through the manifestation of various forms of resistance following the introduction of a new antibiotic for clinical use. The most important factor influencing the emergence and spread of antibiotic resistance is the excessive bacterial exposure to antibiotics [2]. Indiscriminate and over use of antibiotics causes selective pressure, allowing only the fittest genotype to thrive. Despite of the fact that evolution is inevitable, the intensive use of antimicrobial agents in the community, hospital and agriculture is undeniably responsible for fuelling this crisis. Today, bacteria which are resistant not only to a single drug but simultaneously to many drugs are rampantly spread in the community and clinically due to the improper use of antibiotics in the past decade [2, 3]. Antibiotic resistance may result in treatment failure, increased treatment costs as well as the rate of fatalities, and creates even broader infection control problems - spreading resistant bacteria from hospital to community. The persistence of antibiotic resistance urges the need of finding new therapies against the multi-drug resistant bacteria.

*Address correspondence to this author at the School of Pharmacy, Department of Life Sciences, International Medical University, No. 126, Jalan Jalil Perkasa 19, Bukit Jalil, 57000 Kuala Lumpur, Malaysia;

Tel: +60327317578; Fax: +60386567229; Email: erin_lim@imu.edu.my
Since the 1990s, rapid development in molecular biology and high throughput screening has shed light on a more efficient approach to antibiotic discovery. However, the discouraging outcome is that, at present, no antibiotic found by this strategy has yet to enter clinical settings. This is because too much emphasis has been placed on identifying targets and molecules that interact, while too little placed on the actual ability of these molecules to permeate the bacterial cell wall, evade efflux and avoid mutational resistance [4]. Hence discovering a compound that binds to a conserved target does not necessarily equate to finding one with antibiotic activity. Furthermore, antibiotics with a single target are especially vulnerable to mutational resistance.

\section{ATTEMPTS IN NATURAL PRODUCTS}

Previously, natural products screening was almost abandoned, partly because it had ceased to identify new leads, was time consuming and fitted poorly with the changing logistics of high-throughput screening [4]. However, there is resurgence in the use of herbal medicines worldwide. Exploitation of natural products for medicinal uses is a blooming trend nowadays. Natural products are viewed as a privileged group of structures which has evolved to interact with a wide variety of protein targets for specific purposes. Many attempts have been made to investigate the potential role of plant extracts and some active compound for their efficacy to combat the problems of antibiotic resistance in bacteria. Plant extracts consisting complex mixtures of major compounds and their secondary metabolites alongside conventional antibiotics exude possible synergistic effects. The rationale behind the preference for pharmaceutical combina- 
tion is based on a long awareness that many diseases have a complex pathophysiology. Additionally, there are many advantages of using natural products as the antimicrobial compounds such as fewer adverse effects, better patient tolerance, and relatively inexpensive, wide acceptance due to their traditional applications, renewability and better biodegradability.

Many reports have described the emergence of microorganisms that are resistant to different antimicrobial agents, the frequencies and the molecular mechanism of antibiotic resistance involved [2, 3, 5-9]. Extracts from different parts of plants have been widely explored in many studies for their capability in modulating bacterial drug resistance and these studies could serve as reference to provide possible direction for future studies in the reversal of microbial resistance (Table 1). Among all the articles reviewed below, almost half of all the publications did not elucidate the mechanisms of resistance modifying activities, indicating that this missing link should be investigated as it is imperative before natural products can advance into clinical applications. Additionally, very few papers have given insight into the prevalence of the resistance reversibility by natural products, in particular with regards to essential oils. Therefore, the purpose of this mini review is to consolidate all available literature to synthesize fresh insights and to suggest potential future research direction embarking in this area.

\section{Plant Essential Oils}

Plants produce a large array of secondary metabolites as natural protection against microbial and pests attack, as coloring, scent or pollinators attractants. Essential oils, also known as volatile oils, are products of the secondary metabolism of aromatic plants. They are termed "essential" because they represent the very essence and the most important part of the plant. There are distinctive differences between essential oils and crude plant extracts in terms of purity, composition and the process of acquisition. Generally, essential oils are produced through steam distillation or mechanical expression while simple plant extracts often involve the use of solvent such as acetone, ethanol or hexane for extraction. During distillation, water condensate is separated by gravity leaving a very small amount of volatile liquid that is the essential oil. Hence, they are extremely concentrated due to the nature of the extraction process. According to the National Cancer Institute, oils produced by means of chemical solvents are not considered true essential oils as the solvent residues can lead to alteration of the purity and fragrance of the oils [19]. Technically, essential oils are not true oils as they do not contain lipid content instead they are highly complex volatile compounds which consist of about 20-60 components in various concentrations. The components comprise of two biosynthetically related groups namely terpenes and aromatic compounds. In this multi-component mixture, two or three major components are present at relatively high concentrations (20-70\%) compared to other components which are present in trace amounts [20]. For instance, the main component of clove (Syzygium aromaticum) essential oil is eugenol $(68.52 \%)$ while $\alpha$-caryophyllene $(1.85 \%)$ is present in trace amounts [21]. Other major components present in essential oils are terpinen-4-ol (30.41\%) of marjoram (Origanum majorana L.) essential oil, thymol (57.7\%) of Thymus vulgaris essential oil, bicyclogermacrene (26.1\%) and $\beta$-caryophyllene (24.4\%) of Lantana camara $\mathrm{L}$. essential oil, $\alpha$-thujone (41.48\%) of Salvia officinalis L. essential oil, and $\alpha$-(-)-bisabolol (63\%) of Eremanthus erytropappus essential oil [22-26].

Table 1. List of antibiotic resistance modifying plant extracts against a panel of microorganisms.

\begin{tabular}{|c|c|c|c|c|c|}
\hline Plant family name & Part Used & Microorganisms & $\begin{array}{l}\text { Modulation of } \\
\text { Resistance }\end{array}$ & Method of Study & References \\
\hline Rosmarinus officinalis & Aerial part & S. aureus & MDR efflux inhibition & $\begin{array}{c}\text { Ethidium bromide } \\
\text { efflux assay }\end{array}$ & [10] \\
\hline Lycopus europaeus & $\mathrm{N} / \mathrm{A}$ & S. aureus & - & - & [11] \\
\hline Fissistigma cavaleriei & Root & P. aeruginosa & $\begin{array}{l}\text { B-lactamase inhibi- } \\
\text { tion }\end{array}$ & $\begin{array}{l}\text { B-lactamase } \\
\text { inhibitory assay }\end{array}$ & {$[12]$} \\
\hline Cardiospermum grandiflorum & Leaves & S. aureus & - & - & [13] \\
\hline Momordica charantia $\mathbf{L}$. & Leaves & MRSA & $\begin{array}{l}\text { Efflux pump } \\
\text { inhibition }\end{array}$ & $\begin{array}{c}\text { Efflux pump } \\
\text { inhibitory assay }\end{array}$ & [14] \\
\hline Mentha arvensis $\mathrm{L}$. & Leaves & E. coli & - & - & [15] \\
\hline Turnera ulmifolia $\mathrm{L}$. & Leaves & MRSA & - & - & [16] \\
\hline Catha edulis & Leaves & $\begin{array}{l}\text { Streptococcus oralis, } \\
\text { Streptococcus sanguis, Fuso- } \\
\text { bacterium nucleatum }\end{array}$ & - & - & [17] \\
\hline Punica granatum & Fruit & MRSA & Efflux pump inhibiton & $\begin{array}{c}\text { Time-kill assay, } \\
\beta \text {-lactamase production } \\
\text { detection, ethidium } \\
\text { bromide efflux assay }\end{array}$ & [18] \\
\hline
\end{tabular}


Various essential oils have been reviewed to possess different biological properties such as anti-inflammatory, sedative, digestive, antimicrobial, antiviral, antioxidant as well as cytotoxic activities [20, 27]. These findings highlight an exciting scientific interest whereby essential oils warrant special attention because they represent a distinctive group of possible novel drug compounds due to their chemical and structural variance that makes them functionally versatile.

Due to their chemical diversity, the ongoing hypothesis is whether their biological effects are reflected only in the main molecules at the highest levels according to the compositional analysis or that these biological effects arise from the synergism of all molecules present. In most cases reviewed, only the main constituents of certain essential oils such eugenol, thymol and carvacrol were analyzed [28, 29]. Several reports have demonstrated that these compounds exhibited significant antimicrobial activities when tested individually [26, 30]. Dorman and Deans (2000) demonstrated that the individual oil components (mainly with phenolic structures) were able to exhibit a wide spectrum of antibacterial activity and that the chemical structures greatly affect the components effectiveness and their mode of antibacterial action [31]. Bassole et al. (2010) pointed out the synergistic effects on the growth inhibition of Listeria monocytogenes, Enterobacter aerogenes, Escherichia coli and Pseudomonas aeruginosa in eugenol/linalool and eugenol/menthol combinations [32]. Although the biological properties of essential oils are found to be closely related with the major components of the oils, the amplitude of their effects could be attributed to their high concentration comprised in the original oil, masking the effects of minor components or when the high concentration components were isolated and tested alone. Thus, interactive functions of the various components contained in an essential oil, in comparison to the action of one or two main components of the oil seem unresolved. The other side of the coin is that whole essential oils exert greater antibacterial activity compared to the major components alone [27]. It has also been postulated that the function of the main components is regulated by other minor molecules which help in potentiating synergistic effect [32]. It is likely that several components in essential oils play a role in characterizing the fragrance, the density, the texture, the color, ability in cell penetration, lipophilicity, fixation on cell walls, and most importantly the bioavailability. Considering that a vast range of different groups of chemical compounds are present in one essential oil, it is most likely that antibacterial activities cannot be attributed to one specific mechanism or component; and hence, there may be several targets in a cell which result in the potentiating influence. Thus, it is more meaningful and rational to study the whole essential oil rather than some of its components as whether concept of synergism truly exists between the components in essential oils [33].

\section{COMBINATION OF ANTIBIOTICS AND ESSENTIAL OILS TO REVERSE RESISTANCE}

In combination therapy, synergy is said to occur when the combined effect is greater than the sum of the individual effects. An additive effect is observed with the combined effect which is equal to the sum of the individual effects. Indifference is observed when there is no interaction be- tween one another. Antagonism is defined when the combined effect is less than when the two compounds are individually applied. The reversal of resistance is said to occur when a synergistic outcome is observed. As an example, one of the strategies employed was to screen against $\beta$-lactamase producers and by mechanism-based inhibition of the activesite serine hydrolases for compounds that can antagonize the antibiotic-destroying hydrolases. Clavulanic acid (sulbactam or tazobactam) from a streptomycete in combination with amoxicillin was the outcome of this approach [34]. However, the victory against bacterial resistance did not last long; the frequent use of clavulanic acid has led to the emergence of resistant variants like any other of its antibiotic ancestors [35]. As resistant bacterial strains will eventually emerge in response to widespread use of a particular antibiotic and limit its lifetime, knowledge of the principal and specific resistance mechanisms provides scientists the insights into strategies for development of new therapeutics. In the past, when resistance to a $\beta$-lactam antibiotic occurred, pharmaceutical scientists modified the periphery of the $\beta$-lactam warhead to obtain a more effective variant and that was how the second- and third generation $\beta$-lactams of both the penicillin and cephalosporin emerged.

Combination between conventional antimicrobial agents and essential oils is a new concept; a few examples are described (Table 2). Sometimes, essential oils have been found to be synergistic enhancers in that though they may not produce any significant inhibitory effects when used alone, but when they are used in combination with the standard drugs, the combinatory effect surpasses their individual performance and produces enhanced antimicrobial activity [11]. Synergistic activity exerted using essential oils has been found to reduce the minimum effective dose of antibiotics in the treatment of infections. This reduces the adverse effects of the antibiotic. Most importantly, association of antibiotics with essential oils targeting resistant bacteria may have different mechanism of action and it may lead to new choices to overcome the onslaught of microbial resistance. Exploitation of essential oils in preventing bacterial resistance is believed to be more promising because essential oils are multicomponent in nature compared to many conventional antimicrobials that only have a single target site.

Pelargonium graveolens essential oil was reported to reduce the minimum effective dose of norfloxacin against $\mathrm{Ba}$ cillus cereus, Bacillus subtilis, Escherichia coli, and Staphylococcus aureus [30]. Antibiotic modifying capacity of Lantana camara $\mathrm{L}$. essential oil on amikacin against $S$. aureus and $P$. aeruginosa by gaseous contact was demonstrated by Sousa et al. (2012). The microorganisms were exposed to the volatile constituents by indirect contact during the disk diffusion test and the amikacin activity was reported to have increased by $65 \%$ [36]. Rodrigues et al. (2009) reported that the essential oil of Croton zehntneri leaves is able to enhance the gentamicin activity by $42.8 \%$ against $P$. aeruginosa through gaseous contact suggesting that the oil possesses a potential to be used as an adjuvant in antibiotic therapy [37]. These promising investigations indicate that the combination of essential oils with conventional antibiotics provides promising and significant potential for the development of novel therapeutics and treatment of infectious diseases caused by multidrug-resistant microorganisms. There is a need for 
Table 2. List of essential oils/antibiotics combinations showing combinatory effects against a panel of microorganisms.

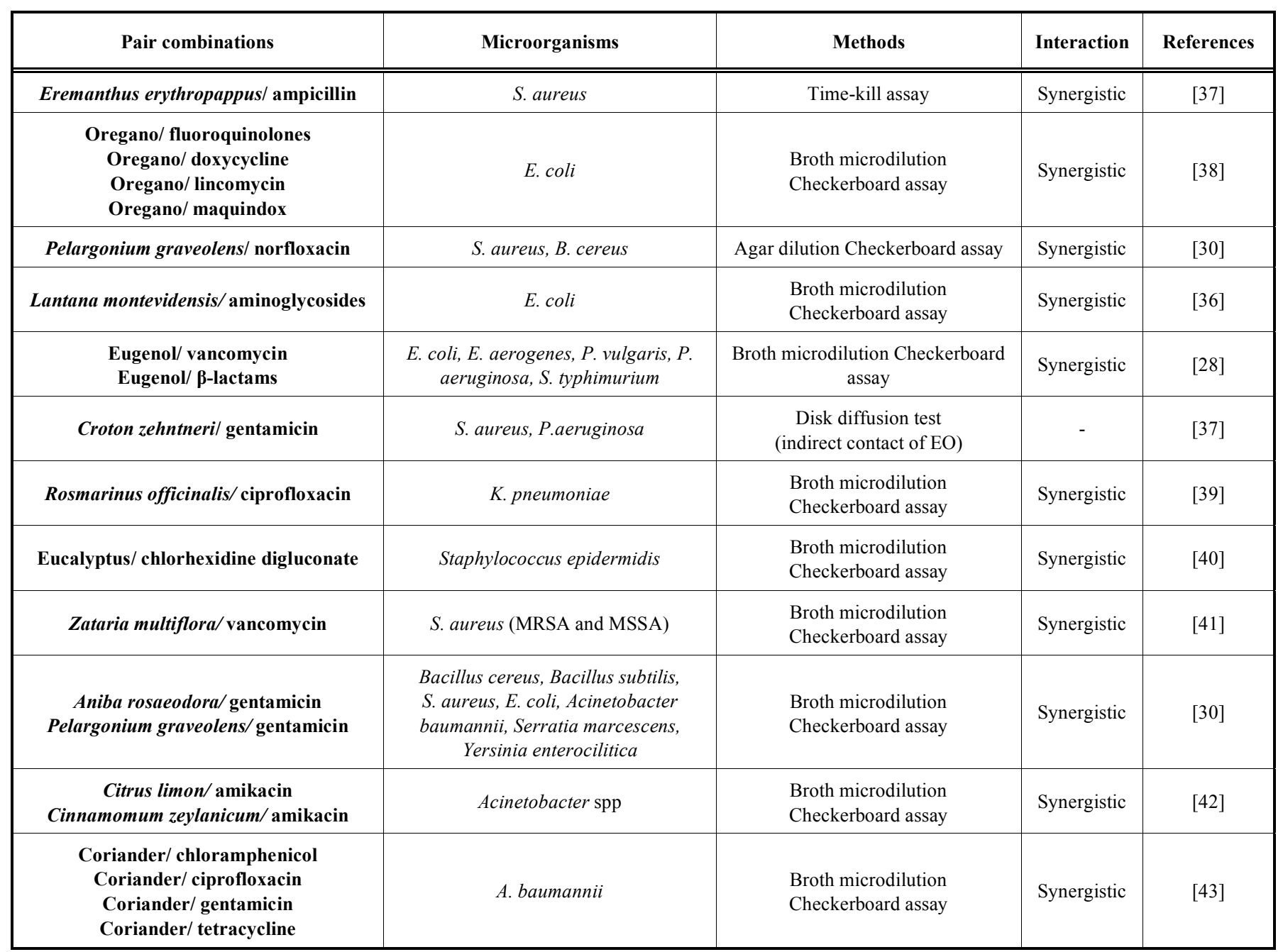

MRSA, methicillin-resistant S. aureus; MSSA, methicillin-sensitive S. aureus.

more studies concerning the molecular basis of synergistic interactions, in order to further understand the synergistic mechanism which is fundamental for this continuing search.

\section{THE PREVALENCE OF RESISTANCE MODIFYING CAPABILITY BY ESSENTIAL OILS}

Antimicrobial agents are classified based on their principal mechanisms of action. These mechanisms include interference with cell wall biosynthesis ( $\beta$-lactams and glycopeptides agents), inhibition of bacterial protein synthesis (marcolides and tetracyclines), interference with nucleic acid synthesis (fluroquinolones and rifampin), inhibition of a metabolic pathway (trimethoprim-sulfamethoxazole, and disruption of bacterial membrane structure (polymyxins and daptomycin) [9]. Three main targets of antibiotics are cell wall, protein and nucleic acids biosynthesis. Throughout the years since antibiotic was introduced, bacteria have acquired various resistances to survive in the deluge of antibiotics. The mechanisms vary and make the work of mitigating the spread of resistance more challenging.

B-lactam antibiotics are the commonest treatment for bacterial infections. This antibiotic class consists of four major groups: penicillins, cephalosporins, monobactams and carbapenems. Hydrolysis of $\beta$-lactam compounds by $\beta$ lactamases is the most widespread mechanism of bacterial resistance against this class of antibiotic. Capability of Staphylococcus aureus in producing penicillinase (a form of $\beta$-lactamase) to destroy penicillin $\mathrm{G}$ was reported within two years after the antibiotic was first introduced [44]. The occurrence of MRSA is a classic example of the redundancy of new antimicrobials with regards to only one species.

\section{Beta-Lactamase Inhibition}

The $\beta$-lactamases are the major defense of gram-negative bacteria against $\beta$-lactam antibiotics. Under the selective pressure of $\beta$-lactams, bacteria produce a vast array of $\beta$ lactamases. Bacteria respond with a plethora of "new" $\beta$ lactamases - including extended-spectrum $\beta$-lactamases (ESBLs), plasmid-mediated AmpC enzymes, and carbapenem-hydrolyzing $\beta$-lactamases (carbapenemases) with variable successes [45]. Resistance is typically mediated by the expression of plasmid-encoded $\beta$-lactamases, such as TEM1, TEM-2, or SHV-1, which hydrolyze and inactivate these drugs. However, investigations into essential oils/antibiotics combination against beta-lactamase producers are currently 
limited, there is one report on the synergistic effects of oregano essential oil in combination with fluroquinolones, doxycycline, lincomycin, maquindox and flofenicol against ESBL-producing $E$. coli suggesting the possibility that essential oils might function as the ESBLs inhibitor [38]

\section{Bacterial Efflux Pump Inhibition}

Most of the antibiotics need to be transported across the cell membrane and subsequently achieve an effective concentration in the cytoplasm to exert inhibitory effects on bacteria. It was demonstrated by Walsh that the overproduction of protein pumps at the bacterial membrane facilitates the pumping out of the drug to be faster than it can diffuse in to keep intra-bacterial drug concentrations below the therapeutic level [8]. The pumps are highly conserved and are chromosomally encoded elements while multidrug resistance efflux pumps are variants of membrane pumps in all microorganisms in response to the external environment. They are able to efflux a large range of compounds including synthetic antibiotics that were not present in the natural ecosystems before their synthesis by humans. Thus, it is believed that bacteria will not easily resist compounds which are natural as compared to the synthetic compounds (the later classes of antibiotics). Related study on phytochemicals addresses this hypothesis and they have been proven to be potent antimicrobial agents. The ability of essential oils in inhibiting the multidrug efflux pump reveals its potential in broader spectrum of pump-inhibitory activity against multi-drug resistant organisms [10]. Lorenzi et al. (2009) evaluated that general component in the essential oil of Helichrysum italicum not only reduces chloramphenicol resistance of the multi-drug resistant Enterobacter aerogens that overexpresses efflux pumps but also modulates the intrinsic resistance of the wild-type control strain and other gram-negative bacteria [46].

\section{Cell Wall and Membrane Disturbance}

The bacterial cell wall biosynthetic machinery remains one of the most promising niches for antibiotic targets. However, the impermeable nature of the gram-negative envelope and presence of multiple efflux pumps in combination with other resistance mechanisms contribute to the difficulty of this task. Clinical resistance to $\beta$-lactams in gram-negative bacteria is often coupled with reduced outer membrane permeability. As the secondary constituents of the aromatic plants, essential oils are known to contain wide ranges of polyphenols and terpenoids. These phenols possess a strong binding affinity to different molecular structures such as protein or glycoproteins due to their large lipophilicity. Hence, they have great affinities for cell membranes and exhibit high potential to permeate through cell walls, leading to the leakage of cell contents [28,47]. The ability of tea tree oil (Melaleuca alternifolia) acting as membrane permeabilizer leading to loss of chemiosmotic control in both grampositive and gram-negative bacteria was elucidated by Cox et al. (2000) [48]. Later, the biological damage of tea tree oil on cell ultra-structures (i.e. cytoplasm and cytoplasmic membrane) was also studied with the aid of electron microscopy $[49,50]$

\section{Anti-Quorum Sensing}

The role of quorum sensing is well known in microbial pathogenicity and antibiotic resistance. Quorum sensing is responsible for motility and swarming patterns, biofilm formation and stress resistance based on the signaling of molecules. An example of a well-studied molecule in this area is acylated homoserine lactones (AHLs). The Gram-negative bacteria use AHLs for signaling whereas the Gram-positive bacteria use modified oligopeptides. The crucial role of quorum sensing on so many essential aspects of the bacterial ecology makes this an interesting process to target to control persistent infections due to antimicrobial resistance.

Screening of potential quorum-quenching activities often involves biosensors and bioluminescene production or inhibition. Some of the common biosensors include Chromobacterium violaceum $\mathrm{CV} 026$ and $\mathrm{N}$-acyl homoserine lactone producing E. coli [51-54]. Rose, geranium, lavender, clove and rosemary oils were found to be the QS inhibitors in the sensor strains Chromobacterium violaceum CV026, Escherichia coli ATTC 31298, Chromobacterium violaceum (CV12472 and CVO26) and Pseudomonas aeruginosa (PAO1) respectively $[52,55]$.

\section{Sensitivity of Gram-Positive and Gram-Negative Bacte- ria Towards Essential Oils}

Generally, essential oils are more efficacious towards gram-positive than gram-negative bacteria [56, 57]. It has been hypothesized that the presence of lipopolysaccharide encompassing the bacterial peptidoglycan layer has restricted the diffusion of hydrophobic compounds into the cytoplasm [58]. However, not all studies of essential oils have concluded that gram-positives are more susceptible [22, 59]. Interestingly, in a study carried out by van Vuuren et al. (2009), combination of the essential oil of Rosmarinus officnalis with ciprofloxacin against gram-positive bacteria gave an antagonistic profile while Rosmarinus officnalis/ciprofloxacin against gram-negative bacteria displayed a favorable synergistic profile [39]. The prevalent antagonistic interaction test against $S$. aureus also suggested that natural therapies using essential oils should be monitored carefully when combined with antibiotics. Since only inadequate sample sets have been studied, more exhaustive investigations would warrant a better potentiating profile of essential oils as resistance modifiers of antibiotics in clinical applications.

\section{STRATEGIES TO BYPASS THE OUTER MEM- BRANE BARRIER IN MULTIDRUG RESISTANT GRAM-NEGATIVE BACTERIA}

In Gram-negative bacteria, the cell envelope comprising an outer and an inner membrane is a sophisticated macromolecule assembly protecting the cell against extracellular toxic compounds. Membrane proteins, namely the porins are involved in regulating the internal accumulation of various hydrophilic molecules including the antibiotics through passive diffusion. The outer membrane of Gram-negative bacteria plays a crucial role in providing an extra layer of protection to the bacteria as a selective barrier. Alterations of composition of outer membrane and porins resulting in reduced permeability in beta-lactam antibiotics have been demonstrated widely in clinical resistant isolates [60]. The existence of large number of antibiotic resistant bacteria species due to this type of mechanism highlights the importance of the outer membrane barrier in antibiotic sensitivity. 
Lipopolysaccharide (LPS) is the major component of the outer membrane of Gram-negative bacteria. LPS contributes greatly to the structural integrity of the bacteria and also increases the negative charge of the cell membrane due to the charged sugars and phosphate present in the polysaccharide [61]. Despite the role of LPS in creating a permeability barrier, the presence of porin proteins in the outer membrane permits passage of molecules smaller than 600 daltons [61, $62]$.

\section{Destabilization of LPS Barrier and Increase of Antibiotic Influx}

As discussed by Bolla et al. (2011), an alternate possibility to facilitate the antibiotics to gain access into the cells is to bypass the outer membrane barrier [63]. To bypass the barrier through LPS modifications, chemical facilitators such as detergents, chaotropic agents and polymyxines have been proposed $[64,65]$. It is noted that only very limited studies have been reported on the molecular basis of the synergy efficacy between antibiotics and the membrane-active agents and also the study parameters.

To circumvent the membrane barrier, destabilization of LPS layer using detergents or chaotropic agents was proposed. Treatment by Tris/EDTA results in massive release of LPS packing and thus facilitates the diffusion of hydrophilic compounds through the membrane lipid barrier. It has been demonstrated that the LPS-disturbed bacteria are more susceptible to antibiotics [66]. However, the major adverse effect of using these chaotropic agents and detergents is their high toxicity on biological membrane, consequently leading to the medical safety issues. Hence, it is valuable to explore the possibility of natural products being potential membrane permeabilizer in restoring the efficacy of the existing antibiotics particularly beta-lactams. As demonstrated by Hurdle et al. (2011), membrane-damaging agents may interfere with multiple targets through the interaction of lipophilic moiety with the bacterial membrane, through alteration of the proton motive force, which in turn leads to leakage of cytoplasmic content and eventually cell death [64].

\section{Cell Targets of the Natural Membrane-Active Agents}

Natural products have been found to have great effects in disrupting the bacterial membrane [67]. It is likely due to the presence of lipophilic compounds such as cyclic hydrocarbons, terpenes and aromatics which are abundantly found in the aromatic plants [68]. Broadly, site(s) of toxic action can be divided into (i) changes in membrane structure, and (ii) changes in membrane function. Specifically, changes in membrane function often involve the changes in energy transduction and changes in activity of membrane-bound enzymes [68].

In the work of Wilson et al. (2001), it is highlighted that the bacterial surface physiology could seek better understanding using available instrumentation through the measurement of zeta potential [69]. The bacteria invest a significant portion of their metabolic energy in the synthesis and maintenance of the components of the cell surface further supporting the idea of interfacial physiology's importance to the general well being of the microorganism [70]. It is vital to understand that the bacterial cell surface is related to the disparate physiological functions such as envelope diffusion, shape maintenance, cell growth and division. It is demonstrated that Gram-negative bacteria have a more negative surface charge than the Gram-positive ones [71]. It is noted that a standardized methodology for evaluating the membrane permeabilizing activity is lacking. Consequently, the activity of the compounds tested in unrelated studies cannot be compared directly. Table 3 summarizes the site of action of essential oils on the membrane.

\section{IN VITRO EVALUATION OF SYNERGY TESTING}

Currently, there is no clear regulation or standardization of the methodology to evaluate the inhibitory activity of essential oils as well-established as for antibiotics [14]. For antibiotics, various methods have been employed by researchers to determine the minimum inhibitory concentration (MIC): disk diffusion test, agar dilution test and broth microdilution test. A number of methods are used to detect synergy as well. The checkerboard and time-kill curve methods are the most widely used techniques and the former is relatively easy to perform and monitor.

The main disadvantage of the results of in vitro studies is that it is difficult to carry out comparison among each of the study because of the different test methods, different methods of extraction of essential oils, test assays, and variation in chemical phytoconstituents in plants due to different agroclimatic conditions, harvesting seasons and plant phenotype. All these factors will influence the phytochemicals present in the essential oils in a considerable manner [15]. In addition, culture conditions have a predominant influence in the studies; and therefore these should be precisely stated in reports.

In vitro susceptibility of a clinical isolate to a particular antibiotic does not guarantee the success of the clinical use of the therapeutic agent. The clinical outcome depends on a wide range of other factors such as the site of infection, pharmacological properties of the antibiotic, concomitance of other diseases and efficiency of specific and non-specific defense mechanism. Thus, in vitro susceptibility testing is necessary but not sufficient for a positive clinical decision.

\section{BACTERIAL RESISTANCE TO ESSENTIAL OILS}

Interest in essential oils as potential therapeutics to eradicate antibiotic resistance has been increasing and the rising concern is whether the bacterial tolerance to the essential oil components would be induced when these compounds are used clinically on a large scale. The extent of bacteria in acquiring resistance to essential oil components has yet to be systematically and extensively investigated. Limited studies have been carried out while much focus has been placed on identifying the novel compound as resistance modifier and expanding the phytopharmaceutical library. Though the tea tree oil has been approved for medicinal use in Australia since 1920s, clinical resistance to the essential oil has not yet been reported [73]. In an in vitro resistance induction study, resistant sub-population of MRSA was detected when the $S$. aureus was repeatedly exposed to tea tree oil for several generations [74]. Conversely, in two other studies using different parameters to study changes in susceptibility of the multiple antibiotic resistance phenotype bacteria, little 
Table 3. Cell targets of membrane-active compounds and its mode of study.

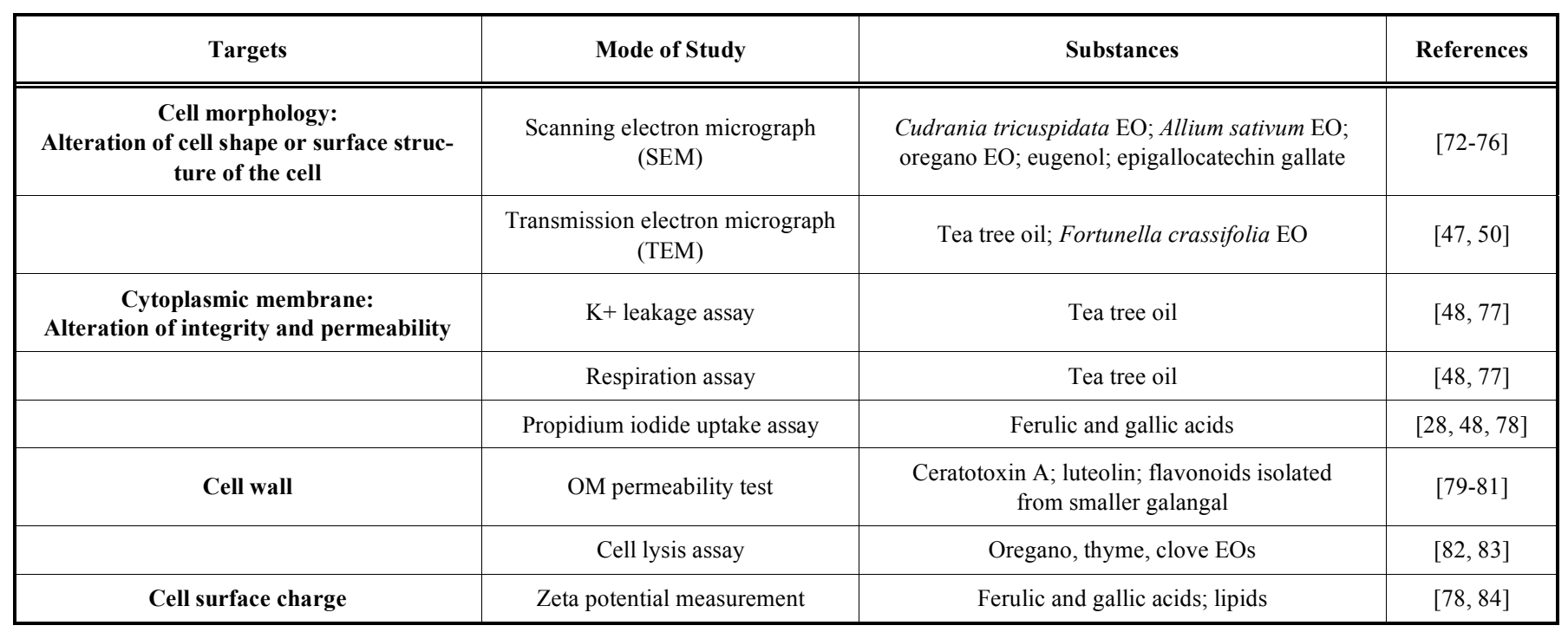

evidence was provided to suggest the occurrence of resistance to tea tree oil $[85,86]$. In a single-step mutant resistant study, mutant resistant to tea tree oil was undetectable at $2 \mathrm{x}$ MIC for the all S. aureus isolates [87]. A similar lack of significantly increased MICs was observed on the development of single- and multistep antibiotic resistance in $S$. aureus and E. coli against tea tree oil [88]. A study involving passaging bacteria up to fifty times with sequential exposure of oregano and cinnamon essential oils was reported. Out of the 48 clinical isolates and 12 reference strains under study, only Serratia marcescens, Morganella morganii, and Proteus mirabilis treated with oregano increased their resistance to this essential oil. No resistance to cinnamon bark oil was reported [89].

Overall, these studies provide limited evidence to suggest the spontaneous occurrence of essential oils resistance. It is likely that the multi-component nature of essential oils may reduce the potential of the occurrence of essential oils resistance because numerous targets need to adapt to hamper the effects of the essential oils. In addition, if membrane permeabilizing effects are one of the modes of action for the essential oil, it is unlikely to that the resistance will develop spontaneously. As discussed by Langeveld et al. (2013), changing membrane structures and/or composition instantaneously arrests the viability of the bacteria [67]. Hurdle et al. (2011) also suggested a low potential for the development of resistance on membrane-active agents due to the LPS modification systems [64]. Issues of potential resistance remain if essential oils make their way into clinical applications, particularly against the multidrug resistant microorganisms.

\section{CONCLUSION}

The reason for research in reversal of antibiotic resistance, broadly, is to preserve a healthy microbial ecosystem surrounding humans. The emergence of antibiotic resistance clearly demonstrates the dynamic evolution of microorganisms' response to the hostile environment of antibiotics. Mastering the evolutionary trajectories of the microbial pathogens would aid in preventing their emergence and dissemination.
As discussed in the review, there are plenty of possibilities for the essential oils to be used in combination with antibiotics as new treatment modalities to the bacterial infections. Despite the promising results given by pharmacological in vitro studies so far, there are problems that still need to be addressed such as stability, selectivity and bioavailability of these natural products in the human body and any possible adverse herb-drug interaction. Additionally, the optimal ratio and dosing regimens should be explored for higher efficacy and decreased toxicity. These critical parameters have to be established in order to provide sufficient evidences to coast through the phases of clinical trials. Or else, new discoveries on bench top, however impressive, will never be translated into drugs for clinical applications.

The exploitation of essential oils as a potential replacement therapy represents 'a new era of phytopharmaceuticals'. Hopefully, the present promising results will open the door for more research into the related field, gain momentum and hasten the process; the discovery will be faster than the evolution of bacteria. The best way is to make full use of the advancement that is available in this era, in the hope that conventional natural products discovery by means of high throughput analysis would shed light on modern drug discovery. Perhaps, in the future, essential oils can progress from being one of the traditional curative agents to become a widely used therapy in the modern medical domain.

\section{CONFLICT OF INTEREST}

The author(s) confirm that this article content has no conflicts of interest.

\section{ACKNOWLEDGEMENTS}

Declared none.

\section{REFERENCES}

[1] World Health Organization. Antimicrobial resistance WHO media centre [updated March 2012; cited 2012 May 5]. Available from: http://www.who.int/mediacentre/factsheets/fs194/en/ 
[2] Canton R, Morosini MI. Emergence and spread of antibiotic resistance following exposure to antibiotics. FEMS Microbiol Rev 2011; 35(5): 977-91.

[3] Neu HC. The crisis in antibiotic resistance. Science 1992; 257(5073): 1064-73.

[4] Livermore DM. Discovery research: the scientific challenge of finding new antibiotics. J Antimicrob Chemother 2011; 66(9): $1941-4$.

[5] Ang JY, Ezike E, Asmar BI. Antibacterial Resistance. Indian J Pediatr 2004; 71: 229-39.

[6] Andersson DI, Hughes D. Persistence of antibiotic resistance in bacterial populations. FEMS Microbiol Rev 2011; 35(5): 901-11.

[7] Cohen ML. Epidemiology of drug resistance: implications for a post-antimicrobial era. Science 1992; 257(5073):1050-5.

[8] Walsh C. Molecular Mechanisms that Confer Antibacterial Drug Resistance. Nature 2000;406:775-81.

[9] Tenover FC. Mechanisms of antimicrobial resistance in bacteria. Am J Med 2006;119(6A): S3-S10.

[10] Oluwatuyi M, Kaatz GW, Gibbons S. Antibacterial and resistance modifying activity of Rosmarinus officinalis. Phytochemistry 2004; 65(24): 3249-54.

[11] Gibbons S, Oluwatuyi M, Veitch NC, Gray AI. Bacterial resistance modifying agents from Lycopus europaeus. Phytochemistry 2003; 62(1): 83-7.

[12] Yang Z, Niu Y, Le Y, Ma X, Qiao C. Beta-lactamase inhibitory component from the roots of Fissistigma cavaleriei. Phytomedicine 2010; 17(2): 139-41.

[13] Nnamani PO, Kenechukwu FC, Oguamanam WN. Cardiospermum grandiflorum leaf extract potentiates amoxocillin activity of Staphylococcus aureus. J Med Plants Res 2012; 6(5): 901-5.

[14] Coutinho HDM, Costa JGM, Falcao-Silva VS, Siqueira-Junior JP, Lima EO. Effect of Momordica charantia L. in the resistance to aminoglycosides in methicillin-resistant Staphylococcus aureus. Comp Immunol Microbiol Infect Dis 2010; 33: 467-71.

[15] Coutinho HD, Costa JG, Lima EO, Falcao-Silva VS, SiqueiraJunior JP. Enhancement of the antibiotic activity against a multiresistant Escherichia coli by Mentha arvensis L. and chlorpromazine. Chemotherapy 2008; 54(4): 328-30.

[16] Coutinho HD, Costa JG, Lima EO, Falcao-Silva VS, Siqueira JP, Jr. Herbal therapy associated with antibiotic therapy: potentiation of the antibiotic activity against methicillin--resistant Staphylococcus aureus by Turnera ulmifolia L. BMC Complement Altern Med 2009; 9: 13.

[17] Al-hebshi N, Al-haroni M, Skaug N. In vitro antimicrobial and resistance-modifying activities of aqueous crude khat extracts against oral microorganisms. Arch Oral Biol 2006; 51(3): 183-8.

[18] Braga LC, Leite AA, Xavier KG, et al. Synergic interaction between pomegranate extract and antibiotics against Staphylococcus aureus. Can J Microbiol 2005 ; 51(7): 541-7.

[19] National Cancer Institute. Aromatherapy and Essential Oils $\left(\mathrm{PDQ}^{\circledR}\right)$ - General Information [updated 10 August 2012; cited $2012 \quad 14 \quad$ July]. Available from: http://www.cancer.gov/cancertopics/pdq/cam/aromatherapy/Health Professional/page2.

[20] Bakkali F, Averbeck S, Averbeck D, Idaomar M. Biological effects of essential oils--a review. Food Chem Toxicol 2008; 46(2): 44675.

[21] Fu YJ, Zu YG, Chen LY, Shi XG, Wang Z, Sun S, Efferth T. Antimicrobial activity of clove and rosemary essential oils alone and in combination. Phytother Res 2007; 21: 989-94.

[22] Busatta C, Vidal RS, Popiolski AS, et al. Application of Origanum majorana L. essential oil as an antimicrobial agent in sausage. Food Microbiol 2008; 25(1): 207-11.

[23] Rota MC, Herrera A, Martinez RM, Sotomayor JA, Jordan MJ. Antimicrobial activity and chemical composition of Thymus vulgaris, Thymus zygis and Thymus hyemalis essential oils. Food Control 2008; 19: 681-7.

[24] Sousa EO, Silva NF, Rodrigues FF, Campos AR, Lima SG, Costa JG. Chemical composition and resistance-modifying effect of the essential oil of Lantana camara Linn. Pharmacogn Mag 2010; 6(22): 79-82.

[25] Alizadeh A, Shaabani M. Essential oil composition, phenolic content, antioxidant and antimicrobial activity in Salvia officinalis L. cultivated in Iran. Adv Environ Biol 2012; 6(1): 221-6.

[26] Nascimento AMA, Brandao MGL, Oliveira GB, Fortes ICP, Chartone-Souza E. Synergistic bactericidal activity of Eremanthus erythropappus oil or b-bisabolene with ampicillin against Staphylococcus aureus. Antonie van Leeuwenhoek 2007; 92: 95100.

[27] Burt S. Essential oils: their antibacterial properties and potential applications in foods--a review. Int J Food Microbiol 2004; 94(3): 223-53.

[28] Hemaiswarya S, Doble M. Synergistic interaction of eugenol with antibiotics against Gram negative bacteria. Phytomedicine 2009; 16(11): 997-1005.

[29] Palaniappan K, Holley RA. Use of natural antimicrobials to increase antibiotic susceptibility of drug resistant bacteria. Int $\mathbf{J}$ Food Microbiol 2010; 140(2-3): 164-8.

[30] Rosato A, Vitali C, De Laurentis N, Armenise D, Antonietta Milillo M. Antibacterial effect of some essential oils administered alone or in combination with Norfloxacin. Phytomedicine 2007; 14(11): 727-32.

[31] Dorman HJ, Deans SG. Antimicrobial agents from plants: antibacterial activity of plant volatile oils. J Appl Microbiol 2000; 88(2): 308-16.

[32] Bassole IH, Lamien-Meda A, Bayala B, et al. Composition and antimicrobial activities of Lippia multiflora Moldenke, Mentha $x$ piperita L. and Ocimum basilicum L. essential oils and their major monoterpene alcohols alone and in combination. Molecules 2010;15(11): 7825-39.

[33] Bassolé IHN, Juliani HR. Essential oils in combination and their antimicrobial properties. Molecules 2012; 17: 3989-4006.

[34] Brown AG. Clavulanic acid, a novel beta-lactamase inhibitor--a case study in drug discovery and development. Drug Des Deliv 1986; 1(1): 1-21.

[35] Blazquez J, Baquero MR, Canton R, Alos I, Baquero F. Characterization of a new TEM-type beta-lactamase resistant to clavulanate, sulbactam, and tazobactam in a clinical isolate of Escherichia coli. Antimicrob Agents Chemother 1993; 37(10): 2059-63.

[36] de Sousa EO, Rodrigues FF, Campos AR, Lima SG, da Costa JG. Chemical composition and synergistic interaction between aminoglycosides antibiotics and essential oil of Lantana montevidensis Briq. Nat Prod Res 2012; 27(10): 942-5.

[37] Rodrigues FF, Costa JG, Coutinho HD. Synergy effects of the antibiotics gentamicin and the essential oil of Croton zehntneri. Phytomedicine 2009; 16(11): 1052-5.

[38] Si H, Hu J, Liu Z, Zeng Z-1. Antibacterial effect of oregano essential oil alone and in combination with antibiotics against extended-spectrum b-lactamase-producing Escherichia coli. FEMS Immunol Med Microbiol 2008; 53: 190-4.

[39] van Vuuren SF, Suliman S, Viljoen AM. The antimicrobial activity of four commercial essential oils in combination with conventional antimicrobials. Lett Appl Microbiol 2009; 48(4): 440-6.

[40] Karpanen TJ, Worthington T, Hendry ER, Conway BR, Lambert PA. Antimicrobial efficacy of chlorhexidine digluconate alone and in combination with eucalyptus oil, tea tree oil and thymol against planktonic and biofilm cultures of Staphylococcus epidermidis. J Antimicrob Chemother 2008; 62(5): 1031-6.

[41] Mahboubi M, Bidgoli FG. Antistaphylococcal activity of Zataria multiflora essential oil and its synergy with vancomycin. Phytomedicine 2010; 17(7): 548-50.

[42] Guerra FQ, Mendes JM, Sousa JP, et al. Increasing antibiotic activity against a multidrug-resistant Acinetobacter spp by essential oils of Citrus limon and Cinnamomum zeylanicum. Nat Prod Res 2011.

[43] Duarte A, Ferreira S, Silva F, Domingues FC. Synergistic activity of coriander oil and conventional antibiotics against Acinetobacter baumannii. Phytomedicine 2012; 19(3-4): 236-8.

[44] Kirby WM. Extraction of a highly potent penicillin inactivator from Penicillin resistant Staphylococci. Science 1944; 99(2579): 452-3.

[45] Jacoby GA, Munoz-Price LS. The new beta-lactamases. N Engl J Med 2005; 352: 380-91.

[46] Lorenzi V, Muselli A, Bernardini AF, et al. Geraniol restores antibiotic activities against multidrug-resistant isolates from gramnegative species. Antimicrob Agents Chemother 2009; 53(5): 2209-11.

[47] Wang YW, Zeng WC, Xu PY, et al. Chemical composition and antimicrobial activity of the essential oil of Kumquat (Fortunella crassifolia Swingle) Peel. Int J Mol Sci 2012; 13: 3382-93. 
[48] Cox SD, Mann CM, Markham JL, et al. The mode of antimicrobial action of the essential oil of Melaleuca alternifolia (tea tree oil). J Appl Microbiol 2000; 88(1): 170-5

[49] Reichling J, Harkenthal M, Geiss H, Hoppe-Tichy T, Saller R. Electron microscopic and biochemical investigations on the antibacterial effects of Australian tea tree oil against Staphylococcus aureus. Curr Top Phytochem 2002; 5: 77-84.

[50] Carson CF, Mee BJ, Riley TV. Mechanism of action of Melaleuca alternifolia (tea tree) oil on Staphylococcus aureus determined by time-kill, lysis, leakage, and salt tolerance assays and electron microscopy. Antimicrob Agents Chemother 2002 ; 46(6): 1914-20.

[51] Krishnan T, Yin WF, Chan KG. Inhibition of quorum sensingcontrolled virulence factor production in Pseudomonas aeruginosa PAO1 by Ayurveda spice clove (Syzygium aromaticum) bud extract. Sensors (Basel) 2012; 12(4): 4016-30.

[52] Khan MS, Zahin M, Hasan S, Husain FM, Ahmad I. Inhibition of quorum sensing regulated bacterial functions by plant essential oils with special reference to clove oil. Lett Appl Microbiol 2009; 49(3): 354-60.

[53] Jaramillo-Colorado B, Olivero-Verbel J, Stashenko EE, WagnerDobler I, Kunze B. Anti-quorum sensing activity of essential oils from Colombian plants. Nat Prod Res 2012; 26(12): 1075-86.

[54] Winson MK, Swift S, Fish L, et al. Construction and analysis of luxCDABE-based plasmid sensors for investigating $\mathrm{N}$-acyl homoserine lactone-mediated quorum sensing. FEMS Microbiol Lett 1998; 163(2): 185-92.

[55] Szabo MA, Varga GZ, Hohmann J, et al. Inhibition of quorumsensing signals by essential oils. Phytother Res 2010; 24(5): 782-6.

[56] Su JY, Zhu L, Tian YJ. Chemical composition and antimicrobial activities of essential oil of Matricaria songarica. Int J Agric Biol 2012; 14: 107-10

[57] Luqman S, Dwivedi GR, Darokar MP, Kalra A, Khanuja SP. Potential of rosemary oil to be used in drug-resistant infections. Altern Ther Health Med 2007; 13(5): 54-9.

[58] Hemaiswarya S, Kruthiventi AK, Doble M. Synergism between natural products and antibiotics against infectious diseases. Phytomedicine 2008; 15(8): 639-52.

[59] Prabuseenivasan S, Jayakumar M, Ignacimuthu S. In vitro antibacterial activity of some plant essential oils. BMC Complement Altern Med 2006; 6: 39.

[60] Pages JM, James CE, Winterhalter $M$. The porin and the permeating antibiotic: a selective diffusion barrier in Gramnegative bacteria. Nat Rev Microbiol 2008 ; 6(12): 893-903.

[61] McGraw-Hill Higher Education (Firm). Annual editions. Microbiology. Annual editions. Dubuque, Iowa: McGraw-Hill Higher Education. p. v.

[62] Delcour AH. Outer membrane permeability and antibiotic resistance. Biochim Biophys Acta 2009; 1794(5): 808-16.

[63] Bolla JM, Alibert-Franco S, Handzlik J, et al. Strategies for bypassing the membrane barrier in multidrug resistant Gramnegative bacteria. FEBS Lett 2011; 585(11): 1682-90.

[64] Hurdle JG, O'Neill AJ, Chopra I, Lee RE. Targeting bacterial membrane function: an underexploited mechanism for treating persistent infections. Nat Rev Microbiol 2011; 9(1): 62-75.

[65] Vooturi SK, Firestine SM. Synthetic membrane-targeted antibiotics. Curr Med Chem 2010; 17(21): 2292-300.

[66] Nikaido H. Molecular basis of bacterial outer membrane permeability revisited. Microbiology and molecular biology reviews : MMBR 2003; 67(4): 593-656.

[67] Langeveld WT, Veldhuizen EJ, Burt SA. Synergy between essential oil components and antibiotics: a review. Crit Rev Microbiol 2013; 40(1): 76-94.

[68] Sikkema J, de Bont JA, Poolman B. Mechanisms of membrane toxicity of hydrocarbons. Microbiol Rev 1995; 59(2): 201-22.

[69] Wilson WW, Wade MM, Holman SC, Champlin FR. Status of methods for assessing bacterial cell surface charge properties based on zeta potential measurements. J Microbiol Methods 2001; 43(3): 153-64.
[70] Beveridge TJ, Graham LL. Surface layers of bacteria. Microbiol Rev 1991; 55(4): 684-705

[71] Sonohara R, Muramatsu N, Ohshima H, Kondo T. Difference in surface properties between Escherichia coli and Staphylococcus aureus as revealed by electrophoretic mobility measurements. Biophys Chem 1995; 55(3): 273-7.

[72] Bajpai VK, Sharma A, Baek K, H. Antibacterial mode of action of Cudrania tricuspidata fruit essential oil, affecting membrane permeability and surface characteristics of food-borne pathogens. Food Control 2013; 32(2): 582-90.

[73] Burt SA, Reinders RD. Antibacterial activity of selected plant essential oils against Escherichia coli O157:H7. Lett Appl Microbiol 2003; 36(3):162-7.

[74] Cho YS, Oh JJ, Oh KH. Synergistic anti-bacterial and proteomic effects of epigallocatechin gallate on clinical isolates of imipenemresistant Klebsiella pneumoniae. Phytomedicine 2011; 18(11): 9416.

[75] Sharma A, Bajpai VK, Baek K, H. Determination of antibacterial mode of action of allium sativum essential oil against foodborne pathogens using membrane permeability and surface characteristic parameters. J Food Safety 2013; 33(2):197-208.

[76] Devi KP, Sakthivel R, Nisha SA, Suganthy N, Pandian SK. Eugenol alters the integrity of cell membrane and acts against the nosocomial pathogen Proteus mirabilis. Arch Pharm Res 2012; 11 : 22

[77] Cox SD, Gustafson JE, Mann CM, et al. Tea tree oil causes K+ leakage and inhibits respiration in Escherichia coli. Lett Appl Microbiol 1998; 26(5): 355-8

[78] Borges A, Ferreira C, Saavedra MJ, Simoes M. Antibacterial activity and mode of action of ferulic and gallic acids against pathogenic bacteria. Microb Drug Resist 2013; 19(4): 256-65.

[79] Marri L, Dallai R, Marchini D. The novel antibacterial peptide ceratotoxin A alters permeability of the inner and outer membrane of Escherichia coli K-12. Curr Microbiol 1996; 33(1): 40-3.

[80] Eumkeb G, Siriwong S, Thumanu K. Synergistic activity of luteolin and amoxicillin combination against amoxicillin-resistant Escherichia coli and mode of action. J Photoch Photobio B, Biology 2012;117: 247-53.

[81] Eumkeb G, Siriwong S, Phitaktim S, Rojtinnakorn N, Sakdarat S Synergistic activity and mode of action of flavonoids isolated from smaller galangal and amoxicillin combinations against amoxicillinresistant Escherichia coli. J Appl Microbiol 2012 ;112(1): 55-64.

[82] Horne D, Holm M, Oberg DG, Chao S, Young DG. Antimicrobial effects of essential oils on Staphylococcus pneumoniae. J Essential Oil Res 2001; 13: 387-92.

[83] Jassim SA, Naji MA. Novel antiviral agents: a medicinal plant perspective. J Appl Microbiol 2003; 95(3): 412-27.

[84] Hamouda T, Baker JR, Jr. Antimicrobial mechanism of action of surfactant lipid preparations in enteric Gram-negative bacilli. J Appl Microbiol 2000; 89(3): 397-403.

[85] Davis AO, O'Leary JO, Muthaiyan A, et al. Characterization of Staphylococcus aureus mutants expressing reduced susceptibility to common house-cleaners. J Appl Microbiol 2005; 98(2): 364-72.

[86] Gustafson JE, Cox SD, Liew YC, Wyllie SG, Warmington JR. The bacterial multiple antibiotic resistant (Mar) phenotype leads to increased tolerance to tea tree oil. Pathology 2001; 33(2): 211-5.

[87] Hammer KA, Carson CF, Riley TV. Frequencies of resistance to Melaleuca alternifolia (tea tree) oil and rifampicin in Staphylococcus aureus, Staphylococcus epidermidis and Enterococcus faecalis. Int J Antimicrob Agents 2008; 32(2): 170-3.

[88] Hammer KA, Carson CF, Riley TV. Effects of Melaleuca alternifolia (tea tree) essential oil and the major monoterpene component terpinen-4-ol on the development of single- and multistep antibiotic resistance and antimicrobial susceptibility. Antimicrob Agents Chemother 2012; 56(2): 909-15.

[89] Becerril R, Nerin C, Gomez-Lus R. Evaluation of bacterial resistance to essential oils and antibiotics after exposure to oregano and cinnamon essential oils. Foodborne Pathogens Disease 2012; 9(8): 699-705 